\title{
Nonlinear $L_{1}$ adaptive control of stagnation pressure in a cryogenic wind tunnel
}

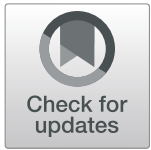

\author{
Rusong Zhu* (D), Mingwei Xie, Daxiong Liao, Gengsheng Tang and Wen Gai
}

\author{
* Correspondence: Rusongzhu@126. \\ com \\ China Aerodynamics Research and \\ Development Center, Mianyang \\ 621000 , China
}

\begin{abstract}
Cryogenic wind tunnel is a sophisticated aerodynamics ground test facility, which operates in cryogenic temperature with injection of liquid nitrogen. The multivariable, nonlinear and coupled dynamics existing between the temperature, pressure and Mach number in the tunnel, poses great challenges for the effective control of the tunnel. $L_{1}$ adaptive control is a new control methodology developed in recent years with good robustness properties, which has good potentials to address these challenges. But this control method does not provide full adaptive feedforward control in its generic structure. In the paper, adaptive feedforward control action is introduced into the standard $L_{1}$ adaptive control architecture for nonlinear systems in the presence of matched un-modeled dynamics. This new control structure is applied to the stagnation pressure control in a cryogenic wind tunnel, which could also be used for the control of temperature and Mach number in the tunnel. This new method could effectively compensate known disturbances with linear gain uncertainty, which occur in the nonlinear systems, while retaining the closed-loop control performance of $L_{1}$ adaptive control. After the proof and discussions on the stability of this method, simulations of the stagnation pressure control in the wind tunnel are presented. The results and analysis demonstrate the effectiveness of the proposed control architecture.
\end{abstract}

Keywords: $L_{1}$ adaptive control, Nonlinear system, Stagnation pressure, Cryogenic wind tunnel

\section{Introduction}

Cryogenic wind tunnel is a sophisticated aerodynamics ground test facility which can provide real flight Reynolds number by operating the wind tunnel in cryogenic temperature with the injection of liquid nitrogen into tunnel circuit [1]. Reynolds number is increased in cryogenic wind tunnel due to the increased gas density and decreased viscosity of the gas at cryogenic temperatures [2]. In contrast with conventional wind tunnels, cryogenic wind tunnel has three main flow parameters, pressure, temperature and Mach number, which should be regulated accurately to obtain high quality flow-field. Due to the interactions between the three variables and their respective control inputs, there exists a multi-variable, nonlinear and coupled dynamics for the three flow states [3]. Thus, a finely designed controller with reasonable robustness and good performance should be deployed in the automatic control of cryogenic wind tunnel to guarantee its smooth operations. At present, some cryogenic wind tunnels with high Reynolds number capabilities have been built in some well-known

(c) The Author(s). 2019 Open Access This article is distributed under the terms of the Creative Commons Attribution 4.0 International License (http://creativecommons.org/licenses/by/4.0/), which permits unrestricted use, distribution, and reproduction in any medium, provided you give appropriate credit to the original author(s) and the source, provide a link to the Creative Commons license, and indicate if changes were made. 
research institutes in the world, such as the DNW-Kryo-Kanal Köln cryogenic wind tunnel in Köln Germany, 0.3-m Transonic Cryogenic Tunnel (TCT) and the National Transonic Facility (NTF) at NASA in the US, the European Transonic Wind tunnel (ETW) in Germany $[4,5]$.

Because of the complex nature of cryogenic wind tunnel dynamics, some studies and researches on the development of control methodology for the tunnel had been carried out from the start of the concept of cryogenic wind tunnel decades ago, mainly concerning the modeling and control of the cryogenic wind tunnel process [6, 7]. Most of the research have been conducted at the TCT, NTF of NASA Langley and ETW in Europe. A nonlinear gain scheduled PI controller with feedforward control was deployed in NTF in its early stages of operation [2]. A control algorithm with self-learning capabilities was designed and implemented in ETW [4]. Since then, some updates and re-innovation of the control system have been reported in recent years [8], while without much details.

The purpose of this paper is to deploy a new adaptive control method with guaranteed transient performance, $L_{1}$ adaptive control [9], to implement the control of cryogenic wind tunnel. Deploying adaptive control in cryogenic wind tunnel has many advantages over using traditional control methods in the tunnel. The main advantage is that it could equip the control system with some self-learning capabilities and would find proper control parameters automatically to achieve desired control performance under uncertainties in the system or model inaccuracies of the tunnel. This would reduce a lot of tuning efforts, time and resources consumptions when the tunnel is in commissioning phase. Rather than considering the control of all three parameters, this paper mainly considers the stagnation pressure control in cryogenic wind tunnel.

Adaptive control was first proposed to address the problem of autopilots in flight control in the mid-1950s [10]. The early development of adaptive control was dominated by experiments, advances made in stability theory later inspired development of the theory for adaptive systems [11]. After that, the interest in adaptive flight control produced many researches on adaptive control in industry and academia. More vibrant researches on adaptive control for aerospace applications appeared at the turn of the century. The driving forces were requirements for reconfiguration and damage control and the desire to simplify extensive and costly verification and validation procedures in flying vehicle development $[12,13] . L_{1}$ adaptive control is one of these results in recent years, a newly developed adaptive control methodology mainly contributed by Hovakimyan and Cao [14-17]. This new adaptive control has some distinguishing features compared with conventional adaptive controls, such as Model Reference Adaptive Control (MRAC). It can be viewed as a modified model reference adaptive control scheme, in which the basic architecture is based on internal model principle [9].

The key feature of $L_{1}$ adaptive control architectures is the guaranteed robustness in the presence of fast adaptation. In this adaptive architecture, the uncertainties in feedback loop can be compensated only within the bandwidth of a filter in its structure [9]. This leads to separation between adaptation and robustness in the adaptive law, and then desired and guaranteed transient performance for the closed-loop systems can be achieved. Details of the theory can be found in [9] and related papers on this issue.

However, the standard $L_{1}$ adaptive control doesn't provide full adaptive feedforward control capacity for known disturbances in systems due to the existence of low-pass 
filter in its control law. Because in normal cases, it is impossible for a control signal coming out of low-pass filter to completely compensate the known disturbances in system. The generic $L_{1}$ adaptive control can compensate some un-modeled or unknown disturbances under specific assumptions about the system [9].

This paper mainly introduces adaptive feedforward control into the standard $L_{1}$ adaptive control for nonlinear system to provide full compensation for known disturbances with linear gain uncertainty. The adaptive feedforward control is based on conventional adaptive scheme, while it is fully integrated into the $L_{1}$ adaptive control structure. Then this new control structure is applied in the stagnation pressure control of a cryogenic wind tunnel.

This paper is organized as follows. Section 2 gives a brief introduction to the facility and the stagnation pressure dynamics of the tunnel. Section 3 presents problem formulation for the $L_{1}$ adaptive control with feedforward control action, and illustrates the controller design and its stability discussion. Section 4 introduces controller design for the stagnation pressure, simulation results and some experimental results, while Section 5 concludes the paper.

\section{Dynamic model of cryogenic wind tunnel}

\subsection{Facility description}

The facility considered in this paper is a closed-circuit, pressurized, outer thermalinsulated small cryogenic wind tunnel, mainly used for some researches related to aerodynamics in cryogenic temperature. The schematic diagram is shown in Fig. 1.

A compressor driven by an electrical motor moves nitrogen gas around the circuit. When in operation, the liquid nitrogen $\left(\mathrm{LN}_{2}\right)$ is injected into the tunnel at the upstream of the compressor and mixed with the gas inside the tunnel. The mass volume of liquid nitrogen injected into the wind tunnel is regulated by $\mathrm{LN}_{2}$ control valve. The gas nitrogen $\left(\mathrm{GN}_{2}\right)$ inside the tunnel is extracted to the $\mathrm{GN}_{2}$ exhaust system by the pressure control valve, which is located at upstream of the stilling chamber. The stagnation (total) pressure is measured at the stilling chamber before test section. The main operation parameters, total pressure, total temperature and Mach number in test section, need to be controlled precisely within the whole operation envelop. The operation range is, $110 \mathrm{~K}$ to $300 \mathrm{~K}$ for total temperature, 1.15 to $4.5 \mathrm{~atm}$ for total pressure, and 0.15 to 1.2 for Mach number respectively.

Normally, the operation of cryogenic wind tunnel starts with a cooling down process with decreasing rate constraint from ambient temperature. After the temperature set

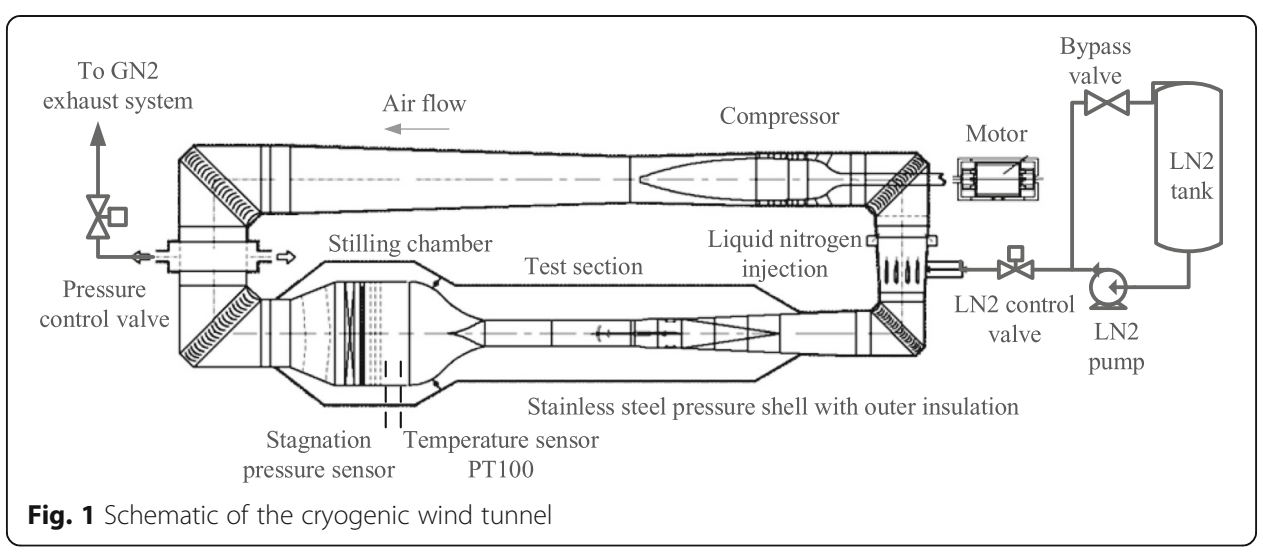


point is reached, quick changes of test section states will be carried out for the test. When the test is finished, a tunnel warming process with rising rate limitation will occur before the end of entire operation.

\subsection{Dynamic model of the cryogenic wind tunnel}

The modeling of the tunnel dynamics in this paper mainly follows the study in reference [2], and the dynamics will be briefed here.

The main state dynamics is given as

$$
\begin{aligned}
& \dot{P}_{t}=\frac{P_{t}}{T_{t}} \cdot T_{t}+\frac{P_{t}}{W_{g}}\left(m_{L}\left(t-\tau_{1}\right)-m_{g}\left(t-\tau_{2}\right)\right) \\
& \dot{T}_{t}=\frac{m_{L}\left(t-\tau_{3}\right)}{W_{g} C_{V}}\left(h_{L}-C_{V} T_{t}\right)+\frac{P_{\text {compressor }}\left(t-\tau_{4}\right)}{W_{g} C_{V}}-\frac{W_{m} C_{m}}{W_{g} C_{V}} \frac{\left(T_{t}-T_{m}\right)}{t_{m}} \\
& \dot{M} a=\left(\frac{N_{f}}{P_{t}^{-0.035} \sqrt{T_{t}}}-k_{1}\right) /\left(k_{2} t_{a}\right)-\frac{M a}{t_{a}}
\end{aligned}
$$

where $P_{t}, T_{t}, M a$ are stagnation pressure, stagnation temperature and Mach number respectively. In (1), $W_{g}$ is gas mass in the tunnel, $m_{L}$ is the liquid nitrogen injection mass flow rate, and $m_{g}$ is the gas nitrogen mass flow blown off from the tunnel. ' $T_{t}$ is the change rate of temperature $T_{t} . \tau_{1}, \tau_{2}, \tau_{3}$ and $\tau_{4}$ are time delays corresponding to each input. (1) is the governing dynamic equation for stagnation pressure in cryogenic wind tunnel. It shows that the stagnation pressure changes are mainly dictated by the blown off mass flow $m_{g}$ and liquid nitrogen injection mass flow $m_{L}$. Equations (2) and (3) denote the stagnation temperature and Mach number dynamics, where $C_{V}$ is specific heat at constant volume $(\mathrm{kJ} / \mathrm{kg} . \mathrm{K}), C_{m}$ is specific heat of metal tunnel wall $(\mathrm{kJ} / \mathrm{kg} . \mathrm{K}), h_{L}$ is heat of the vaporization of liquid nitrogen $(\mathrm{kJ} / \mathrm{kg}), P_{\text {compressor }}$ is power consumed by the compressor, $W_{m}$ is mass of metal wind tunnel wall $(\mathrm{kg}), T_{m}$ is metal tunnel wall temperatures $(\mathrm{K}), N_{f}$ is compressor speed (RPM), $k_{1}$ and $k_{2}$ are constants specific to the facility, and $t_{a}$ is plenum time constant of the tunnel (s). The detailed meanings of (2) and (3) for stagnation temperature and Mach number dynamics can be found in reference [2], and will be omitted here.

These equations of dynamics clearly show that there is obvious coupled and nonlinear behavior of the cryogenic wind tunnel process.

\section{Nonlinear $L_{1}$ adaptive control with adaptive feedforward control}

\subsection{Problem formulation}

Consider a nonlinear (and time-varying) dynamical system described by equations,

$$
\begin{aligned}
& \dot{x}(t)=A_{m}(t) x(t)+b(t)\left(\mu(t)+f(t, x(t), z(t))+u_{F}(t)\right) \\
& x(0)=x_{0} \\
& y(t)=c^{T} x(t) \\
& \dot{x}_{z}(t)=g\left(t, x_{z}(t), x(t)\right), x_{z}(0)=x_{z 0} \\
& z(t)=g_{0}\left(t, x_{z}(t)\right), x_{z}(0)=x_{z 0}
\end{aligned}
$$

where $x(t) \in R^{n}$ is the system state and measurable, $A_{m}(t) \in R^{n \times n}$ and $b(t) \in R^{n}$ are known matrix and control gain vector respectively. (Note: A known $A_{m}(t)$ represents the desired controlled closed-loop system behavior. This will not pose a problem in 
describing a real system where there may exist many uncertainties. Because for some systems, these uncertainties could be counted by partially known or unknown terms like $f(t, x(t), z(t)), z(t)$ and $x_{z}(t)$ in (4).) $c \in R^{n}$ is a known constant vector and $y(t) \in R$ is the system output. The $f: R \times R^{n} \times R^{l} \rightarrow R$ is an unknown nonlinear map representing the matched nonlinear uncertainties. $x_{z}(t) \in R^{m}$ and $z(t) \in R^{l}$ are the state and output of the un-modeled dynamics. $g: R \times R^{m} \times R^{n} \rightarrow R^{m}, g_{0}: R \times R^{m} \rightarrow R^{l}$ are unknown nonlinear maps. $u_{F}(t) \in R$ is the known or measurable disturbance in the input channel. $\mu(t) \in R$ is the output of system:

$$
\mu(s)=F(s) u(s)
$$

where $u(t) \in R$ (for $u(s)$ ) is the control signal, and $F(s)$ is an unknown BIBO-stable and proper transfer function with known sign of its DC gain. Normally the $F(s)$ is used to represent the actuator dynamics in system, which cannot be exactly known. The initial condition $x_{0}$ is assumed to be inside a known set, $\left\|x_{0}\right\|_{\infty}<\rho_{0}<\infty$, with known $\rho_{0}>0$. In addition, let's define $X \triangleq\left[x^{T}, z^{T}\right]^{T}$.

Remark 1 The main difference between (4) and the standard form of (5.25) in section 5.2 of reference [9], lies in the extra known disturbance term $u_{F}(t)$ in (4). This is used to represent known disturbance in the control input channel. An adaptive feedforward control scheme is designed later to cancel this known disturbance. The $L_{1}$ adaptive controller acts as the feedback part of the whole control law to stabilize the system (4) with desired closed-loop system response.

For the problem to be properly addressed, the following assumptions are imposed for the system (4). Most of the assumptions follow that in [9].

Assumption 1 (Uniform Asymptotic Stability of Desired System) The matrix $A_{m}(t)$ is continuously differentiable. For $t \geq 0,\left\|A_{m}(t)\right\|_{\infty} \leq \mu_{1},\left\|A_{m}(t)\right\|_{\infty} \leq \mathrm{d}_{A}$, and $\operatorname{Re}\left[\lambda_{i}\left(A_{m}(t)\right)\right] \leq-\mu_{2} \forall \mathrm{i}=1, \ldots, \mathrm{n}$, where $\mu_{1}, \mu_{2}$ and $\mathrm{d}_{A}$ are positive constants, and $\lambda_{i}\left(A_{m}(t)\right)$ is a pointwise eigenvalue of $A_{m}(t)$. Further, for all $\mathrm{t} \geq 0$, the equilibrium of the state equation

$$
\dot{x}(t)=A_{m}(t) x(t) x\left(t_{0}\right)=x_{0}
$$

is exponentially stable, and the solution of

$$
A_{m}^{T}(t) P(t)+P(t) A_{m}(t)=I
$$

satisfies $\|\cdot P(t)\|_{\infty}<1$.

Assumption 2 (Uniform boundedness of $\mathbf{b}(\mathbf{t})$ and its derivative) There exist positive constants $\mu_{b}, \mathrm{~d}_{b}>0$, such that $\|\boldsymbol{b}(\boldsymbol{t})\|<\mu_{b},\|\cdot \boldsymbol{b}(\boldsymbol{t})\|<\mathrm{d}_{b}$.

Assumption 3 The pair $\left(A_{m}(t), \mathrm{b}(t)\right)$ is strongly controllable.

Assumption 4 (Uniform boundedness of $\boldsymbol{f}(\boldsymbol{t}, \mathbf{0}, \mathbf{0})$ ) There exists $B>0$, such that $|f(t, 0,0)|<B$ holds for all $\mathrm{t} \geq 0$.

Assumption 5 (Semi-global uniform boundedness of partial derivatives) For arbitrary $\delta>0$, there exist positive constants $d_{f x}(\delta)>0$ and $d_{f t}(\delta)>0$ independent of time, such that for all $\|X\|_{\infty}<\delta$, the partial derivatives of $\mathrm{f}(t, x, z)$ are piecewisecontinuous and bounded, 


$$
\left\|\frac{\partial f(t, x, z)}{\partial(x, z)}\right\|_{1} \leq d_{f x}(\delta)\left|\frac{\partial f(t, x, z)}{\partial t}\right| \leq d_{f t}(\delta)
$$

Assumption 6 (Stability of un-modeled dynamics) The $x_{z}$ dynamics are BIBO stable with respect to both initial conditions $x_{z 0}$ and input $x(t)$, there exist $C_{1}, C_{2}>0$ such that for all $t \geq 0$

$$
\left\|z_{t}\right\|_{L_{\infty}} \leq C_{1}\left\|x_{t}\right\|_{L_{\infty}}+C_{2}
$$

Assumption 7 (Partial knowledge of actuator dynamics) There exists $C_{F}>0$ verifying $\|F(s)\|_{L_{1}}<C_{F}$. Also, it is assumed that there exist known constants $\omega_{l}, \omega_{u} \in \mathrm{R}$ satisfying

$$
0<\omega_{l} \leq F(0) \leq \omega_{u}
$$

where, without loss of generality, it is assumed $F(0)>0$.

Assumption 8 (Boundedness of $\boldsymbol{u}(\boldsymbol{t})$ and $\boldsymbol{u}_{\boldsymbol{F}}(\boldsymbol{t})$ ) The control signal $u(t)$ and known disturbance $u_{F}(t)$ are continuous, and moreover, there exist positive constants $\rho, \rho_{u}, \rho^{\cdot} u, \rho_{u_{F}}, \rho^{\cdot} u_{F}$, the following bounds hold

$$
\begin{aligned}
& \left\|x_{\tau}\right\|_{L_{\infty}} \leq \rho, \\
& \left\|u_{\tau}\right\|_{L_{\infty}} \leq \rho_{u},\left\|\dot{u}_{\tau}\right\|_{L_{\infty}} \leq \rho_{\dot{u}} \\
& \left\|u_{F \tau}\right\|_{L_{\infty}} \leq \rho_{u_{F}},\left\|\dot{u}_{F \tau}\right\|_{L_{\infty}} \leq \rho_{u_{F}}
\end{aligned}
$$

3.2 Nonlinear $L_{1}$ adaptive control architecture with feedforward control action

Controller design The controller design for nonlinear system (4) follows the main $L_{1}$ adaptive control structure presented in section 5.2 of reference [9]. However, because of the existence of known disturbance $u_{F}(t)$ in (4), the control signal is expressed as

$$
u(t)=u_{f f}(t)+u_{f d}(t)
$$

where $u_{f f}(t)$ is the feedforward control signal to cancel the disturbance $u_{F}(t)$ in (4), and $u_{f d}(t)$ is the feedback control signal provided by the $L_{1}$ adaptive control loop. Although the control signal $u_{f f}(t)$ is different from the $u_{f d}(t)$, it is still derived from the framework of $L_{1}$ adaptive control, which will be introduced in the following. This new $L_{1}$ adaptive control structure with feedforward control loop is shown in Fig. 2, where the parts in the dashed line box are the standard $L_{1}$ adaptive control loop.

\section{State predictor}

The state predictor is given as

$$
\begin{aligned}
& \dot{\hat{x}}(t)=A_{m} \hat{x}(t)+b(t)\left(\hat{\omega}_{F}(t) u_{F}(t)+u_{f f}(t)+\hat{\omega}_{p v}(t) u_{f d}(t)+\hat{\theta}(t)\left\|x_{t}\right\|_{L_{\infty}}+\hat{\sigma}(t)\right) \\
& \hat{x}(0)=x_{0} \\
& \hat{y}(t)=c^{T} \hat{x}(t)
\end{aligned}
$$

where $\hat{x}(t) \in R^{n}$ is the predictor state, and $\hat{\omega}_{p v}$ is the estimate for control gain uncertainty, which could cover the gain uncertainty in the control input channel and unknown actuator dynamics. $\hat{\omega}_{F}$ is the estimate for feedforward control gain uncertainty. $\hat{\theta}(t)$ is the 


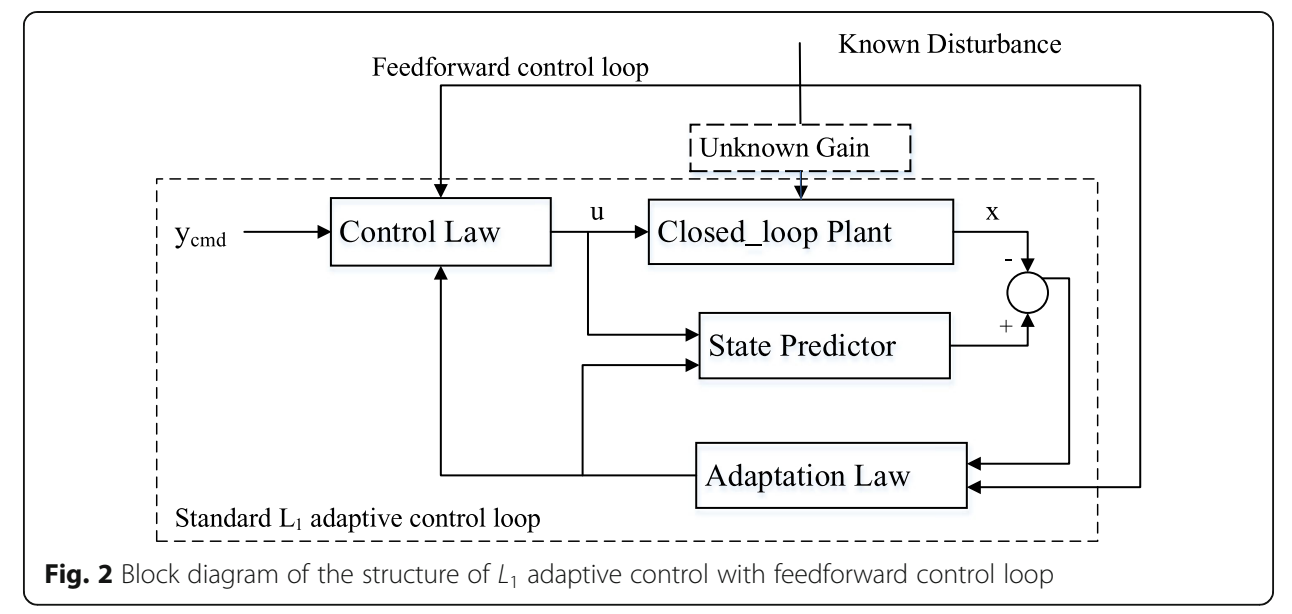

main parameter estimate for the nonlinearity in the system, and the estimate of $\hat{\sigma}(t)$ could represent some un-modeled dynamics and disturbance in the system.

Remark 2 The two terms $\hat{\omega}_{F}(t) u_{F}(t)$ and $u_{f f}(t)$ in (8), which are absent in the standard form of state predictor in [9], are used to form the adaptive law and control law for the disturbance signal $u_{F}(t)$ in the system (4).

\section{Adaptation Laws}

The adaptation laws are given as

$$
\begin{array}{ll}
\dot{\hat{\omega}}_{p v}(t)=\Gamma \operatorname{Proj}\left(\hat{\omega}_{p v}(t),-\tilde{x}^{T}(t) P(t) b(t) u_{f d}(t)\right) & \hat{\omega}_{p v}(0)=\hat{\omega}_{p v 0} \\
\dot{\hat{\omega}}_{F}(t)=\Gamma_{F} \operatorname{Proj}\left(\hat{\omega}_{F}(t),-\tilde{x}^{T}(t) P(t) b(t) u_{F}(t)\right) & \hat{\omega}_{F}(0)=\hat{\omega}_{F 0} \\
\dot{\hat{\theta}}(t)=\Gamma \operatorname{Proj}\left(\hat{\theta}(t),-\tilde{x}^{T}(t) P(t) b(t)\left\|x_{t}\right\|_{L_{\infty}}\right) & \hat{\theta}(0)=\hat{\theta}_{0} \\
\dot{\hat{\sigma}}(t)=\Gamma \operatorname{Proj}\left(\hat{\sigma}(t),-\tilde{x}^{T}(t) P(t) b(t)\right) & \hat{\sigma}(0)=\hat{\sigma}_{0}
\end{array}
$$

where $\tilde{x}(t)=\hat{x}(t)-x(t)$ is the prediction error, $\Gamma$ is the adaptation gain for the $L_{1}$ adaptive control loop, and $\Gamma_{F}$ is the adaptation gain for the $\hat{\omega}_{F} . P(t)$ is defined in assumption 1. The projection operator $\operatorname{Proj}()$ ensures that all estimates of these parameters are bounded.

Remark 3 The feedforward gain estimation $\hat{\omega}_{F}$ is used as the feedforward control gain in $u_{f f}(t)$ in (7) due to the control gain uncertainty of $\omega_{p v}$. For the system here, there should be $\omega_{F}=\omega_{p v}$. But for the convenience of design, an over-parameterization strategy is used. A separate different $\omega_{F}$ is used to represent the gain uncertainty of the disturbance $u_{F}(t)$. In addition, the adaptive law for $\hat{\omega}_{F}(t)$ is independent, and not a part of the standard $L_{1}$ adaptive law, thus the adaptive gain $\Gamma_{F}$ is different from the $\Gamma$ of $L_{1}$ adaptive law.

\section{Control Law}

As stated in (7), the control law consists two components

$$
\begin{aligned}
& u(t)=u_{f f}(t)+u_{f d}(t) \\
& u_{f f}(t)=-\hat{\omega}_{F}(t) u_{F}(t) \\
& u_{f d}(s)=-k D(s)\left(\hat{\eta}(s)-r_{g}(s)\right)
\end{aligned}
$$

where the $r_{g}(s)$ and $\hat{\eta}(s)$ are the Laplace transforms of $r_{g}(t)=k_{g}(t) r(t)$ and $\hat{\eta}(t)=\hat{\omega}_{p v}(t) u_{f d}$ 
$(t)+\hat{\theta}(t)\left\|x_{t}\right\|_{L_{\infty}}+\hat{\sigma}(t)$ respectively, while $k_{g}(t)=-1 /\left(c^{T} A_{m}{ }^{-1}(t) b(t)\right)$ is the feedforward gain required for tracking the reference signal $r(t) . k>0$ in (10) is the positive feedback gain in the filtering structure, and $D(s)$ is a strictly proper stable transfer function, which would lead to a strictly proper stable filter

$$
C(s)=\frac{k F(s) D(s)}{1+k F(s) D(s)}
$$

with DC gain $C(0)=1$.

Remark 4 Because the $u_{F}(t)$ should be compensated by the feedforward control signal $u_{f f}(t)$ in real-time, thus, the control signal $u_{f f}(t)$ can not pass through a filter like that in the feedback control law $u_{f d}(t)$. Otherwise, the adaptive law for $\hat{\omega}_{F}$ in (9) cannot guarantee the stability of the control law in (10).

The feedback control law $u_{f d}(t)$ is realized by a recursive structure, which is shown in Fig. 3.

Remark 5 The equations (8) (10) constitute the nonlinear $L_{1}$ adaptive controller with feedforward control action for the system (4), subjected to a $L_{1}$ norm condition defined in the section 5.2.2 in [9]. The main difference of the controller from the standard $L_{1}$ adaptive control law for nonlinear system lies in the extra adaptive feedforward part in (8) (10). The stability analysis and some proof are introduced next.

\subsection{Stability analysis of the control law}

\subsubsection{Equivalent linear time-varying system for nonlinear dynamics}

According to the study in section 5.2 in reference [9], the original nonlinear system (4) with un-modeled dynamics can be transformed into an equivalent (semi-) linear timevarying system with unknown time-varying parameters and disturbances under the assumptions on the signals of the system in part 3.1 of this section.

From the system (4) and the bounds in (6), it follows that $\left\|x_{\tau}\right\|_{L_{\infty}}$ is bounded for all $\tau \in[0, \infty)$.

According to the Lemma A.9.1 in [9], there exist continuous $\theta(t)$ and $\sigma_{1}(t)$ with (piecewise)-continuous derivatives, defined over $\mathrm{t} \in[0, \tau]$ such that

$$
\begin{aligned}
& |\theta(t)|<L_{\rho},|\dot{\theta}(t)|<d_{\theta} \\
& \left|\sigma_{1}(t)\right|<\Delta_{1},\left|\dot{\sigma}_{1}(t)\right|<d_{\sigma_{1}}
\end{aligned}
$$

where $L_{\rho}, \Delta_{1}, d_{\theta}, d_{\sigma_{1}}>0$. Then the nonlinear function $f(t, x(t), z(t))$ in (1) can be expressed as

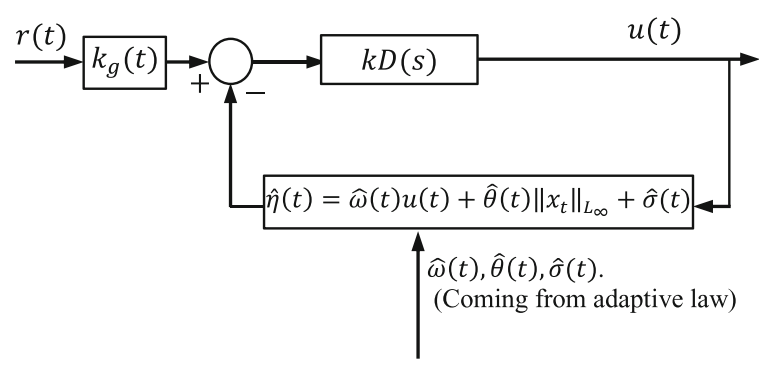

Fig. 3 Structure of the recursive feedback control law 


$$
f(t, x(t), z(t))=\hat{\theta}(t)\left\|x_{t}\right\|_{L_{\infty}}+\sigma_{1}(t) .
$$

Using the assumption 8 and the lemma A.10.1 in [9], the signal $\mu(t)$ in (4) can be rewritten as

$$
\mu(t)=\omega_{p \nu} u(t)+\sigma_{2}(t)
$$

where $\omega_{p v} \in\left(\omega_{l}, \omega_{u}\right)$ is an unknown constant and $\sigma_{2}(t)$ is a continuous signal with (piecewise)-continuous derivative, defined over $\mathrm{t} \in[0, \tau]$, such that

$$
\left|\sigma_{2}(t)\right|<\Delta_{2},\left|\dot{\sigma}_{2}(t)\right|<d_{\sigma_{2}}
$$

where $\Delta_{2}, d_{\sigma_{2}}>0$.

Then the system (4) can be rewritten over $\mathrm{t} \in[0, \tau]$ as

$$
\begin{aligned}
& \dot{x}(t)=A_{m}(t) x(t)+b(t)\left(\hat{\omega}_{F}(t) u_{F}(t)+u_{f f}(t)+\omega_{p v} u_{f d}(t)+\theta(t)\left\|x_{t}\right\|_{L_{\infty}}+\sigma(t)\right) x(0)=x_{0} \\
& y(t)=c^{T} x(t)
\end{aligned}
$$

where $\hat{\sigma}(t)=\sigma_{1}(t)+\sigma_{2}(t)$ is the unknown signal subject to $|\sigma(t)|<\Delta,|\sigma(t)|<d_{\sigma}$, with $\Delta=\Delta_{1}+\Delta_{2}$ and $d_{\sigma}=d_{\sigma_{1}}+d_{\sigma_{2}}$. Additionally, $\omega_{F} \in \Omega_{F}=\left(\omega_{F l}, \omega_{F u}\right), \omega_{p v} \in \Omega_{p v}=\left(\omega_{l}\right.$, $\left.\omega_{u}\right), \theta(t) \in \Theta$, where $0<\omega_{F l}<\omega_{F u}, 0<\omega_{l}<\omega_{u}$ are known upper and lower bounds, $\Theta$ is a known convex compact set, and $\Delta \in R^{+}$is a known (conservative) bound of $\sigma(t)$.

Remark $6 \hat{\omega}_{F}(t)$ in (11) is introduced by the feedforward gain uncertainty of $u_{f f}(t)$ due to the control gain uncertainty of the system. However, for convenience of the control law derivation process, the feedforward gain uncertainty $\hat{\omega}_{F}(t)$ is placed with $u_{F}(t)$, instead of $u_{f f}(t)$ in (11). The equations in state predictor of (8) follow the form of the equivalent equations (11) for the original system (4).

\subsubsection{Transient and steady-state performance}

With the system dynamics as (11), the error dynamics between the state predictor (8) and the system is given by

$$
\dot{\tilde{x}}(t)=A_{m} \tilde{x}(t)+b(t)\left(\tilde{\omega}_{F}(t) u_{F}(t)+\tilde{\omega}_{p v}(t) u_{f d}(t)+\tilde{\theta}(t)\left\|P_{t}\right\|_{L_{\infty}}+\tilde{\sigma}(t)\right) \tilde{x}(0)=0
$$

where $\tilde{x}(t)=\hat{x}(t)-x(t), \quad \tilde{\omega}_{F}=\hat{\omega}_{F}-\omega_{F}, \quad \tilde{\omega}_{p v}=\hat{\omega}_{p v}(t)-\omega_{p v}, \tilde{\theta}(t)=\hat{\theta}(t)-\theta(t)$, and $\tilde{\sigma}(t)$ $=\hat{\sigma}(t)-\sigma(t)$. Then, for the transient performance of the control law, we have a lemma similar to the lemma 5.2.2 in [9], but with the additional bounded term related to the signal $u_{F}(t)$.

Lemma 1 For the system (8) and the controller defined by equations $(8) \sim(10)$, with the assumptions 1 to 8 in part 3.1 of this section verified, then

$$
\left\|\tilde{x}_{\tau}\right\|_{L_{\infty}} \leq \sqrt{\frac{\theta_{m}\left(\rho, \rho_{u}, \rho_{u_{u}}, \rho_{u_{F}}, \rho_{u_{F}}\right)}{\lambda_{\min }(P) \Gamma}}+\sqrt{\frac{k_{m}\left(\omega_{F u}-\omega_{F l}\right)^{2}}{\lambda_{\min }(P) \Gamma_{F}}}
$$

where $\theta_{m}\left(\rho, \rho_{u}, \rho \cdot{ }_{u}, \rho_{u_{F}}, \rho u_{u_{F}}\right)=\left(\omega_{u}-\omega_{l}\right)^{2}+4 L_{\rho}^{2}+4 \Delta^{2}+4 \frac{\lambda_{\max }(P)}{1-\epsilon_{P}}\left(L_{\rho} d_{\theta}+\Delta d_{\sigma}\right)$, and $0 \leq \epsilon_{P}<1, \lambda_{\min }(P)$ and $\lambda_{\max }(P)$ are the minimum and maximum eigenvalue of matrix $\mathrm{P}(\mathrm{t})$ respectively. $k_{m}$ is a specific positive constant.

Proof: Consider the following Lyapunov function candidate 
$V\left(\tilde{x}, \tilde{\omega}_{F}, \tilde{\omega}_{p \nu}, \tilde{\theta}, \tilde{\sigma}\right)=\tilde{x}^{T}(t) P(t) \tilde{x}(t)+\frac{1}{\Gamma_{F}} \tilde{\omega}_{F}{ }^{2}(t)+\frac{1}{\Gamma}\left(\tilde{\omega}_{p \nu}{ }^{2}(t)+\tilde{\theta}^{2}(t)+\tilde{\sigma}^{2}(t)\right)$

Using the adaptation laws in (9), the derivative of the Lyapunov function candidate $V$ along the trajectory of the system (12) is computed as

$$
\begin{aligned}
& \dot{V}\left(\tilde{x}, \tilde{\omega}_{F}, \tilde{\omega}_{p v}, \tilde{\theta}, \tilde{\sigma}\right)=\tilde{x}^{T}(t)\left(P A_{m}+A_{m}{ }^{T} P+\dot{P}(t)\right) \tilde{x}(t)+2 \tilde{x}^{T}(t) P(t) b(t) \tilde{\omega}_{F}(t) u_{F}(t) \\
& +2 \tilde{x}^{T}(t) P(t) b(t) \tilde{\omega}_{p v} u_{f d}(t)+2 \tilde{x}^{T}(t) P(t) b(t) \tilde{\theta}(t)\left\|x_{t}\right\|_{L_{\infty}}+2 \tilde{x}^{T}(t) P(t) b(t) \tilde{\sigma}(t) \\
& +\frac{2}{\Gamma_{F}} \tilde{\omega}_{F}(t) \dot{\hat{\omega}}_{F}(t)+\frac{2}{\Gamma}\left(\tilde{\omega}_{p v}(t) \dot{\hat{\omega}}_{p v}(t)+\tilde{\theta}(t) \dot{\hat{\theta}}(t)+\tilde{\sigma}(t) \dot{\hat{\sigma}}(t)\right)-\frac{2}{\Gamma}(\tilde{\theta}(t) \dot{\theta}(t)+\tilde{\sigma}(t) \dot{\sigma}(t)) \\
& =-\tilde{x}^{T}(t)(1-\dot{P}(t)) \tilde{x}(t)-\frac{2}{\Gamma}(\tilde{\theta}(t) \dot{\theta}(t)+\tilde{\sigma}(t) \dot{\sigma}(t)) \\
& +2 \tilde{\omega}_{F}(t)\left(-\tilde{x}^{T}(t) P(t) b(t) u_{F}(t)+\frac{1}{\Gamma_{F}} \dot{\hat{\omega}}_{F}(t)\right) \\
& +2 \tilde{\omega}_{p v}(t)\left(\tilde{x}^{T}(t) P(t) b(t) u_{f d}(t)+\frac{1}{\Gamma} \dot{\hat{\omega}}_{p v}(t)\right) \\
& +2 \tilde{\theta}(t)\left(\tilde{x}^{T}(t) P(t) b(t)\left\|x_{t}\right\|_{L_{\infty}}+\frac{1}{\Gamma} \cdot \hat{\theta}(t)\right)+2 \tilde{\sigma}(t)\left(\tilde{x}^{T}(t) P(t) b(t)+\frac{1}{\Gamma} \hat{\sigma}(t)\right) \\
& =-\tilde{x}^{T}(t)(1-\dot{P}(t)) \tilde{x}(t)-\frac{2}{\Gamma}(\tilde{\theta}(t) \dot{\theta}(t)+\tilde{\sigma}(t) \dot{\sigma}(t)) \\
& +2 \tilde{\omega} \tilde{\omega}_{F}(t)\left(-\tilde{x}^{T}(t) P(t) b(t) u_{F}(t)+\operatorname{Proj}\left(\hat{\omega}_{F}(t), \tilde{x}^{T}(t) P(t) b(t) u_{F}(t)\right)\right) \\
& +2 \tilde{\omega}_{p v}(t)\left(\tilde{x}^{T}(t) P(t) b(t) u_{f d}(t)+\operatorname{Proj}\left(\hat{\omega}_{p v}(t),-\tilde{x}^{T}(t) P(t) b(t) u_{f d}(t)\right)\right) \\
& +2 \tilde{\theta}(t)\left(\tilde{x}^{T}(t) P(t) b(t)\left\|x_{t}\right\|_{L_{\infty}}+\operatorname{Proj}\left(\hat{\theta}(t),-\tilde{x}^{T}(t) P(t) b(t)\left\|x_{t}\right\|_{L_{\infty}}\right)\right) \\
& +2 \tilde{\sigma}(t)\left(\tilde{x}^{T}(t) P(t) b(t)+\operatorname{Proj}\left(\hat{\sigma}(t),-\tilde{x}^{T}(t) P(t) b(t)\right)\right)
\end{aligned}
$$

Using the property of the operator $\operatorname{Proj}()[9,18]$, there exist

$$
\begin{aligned}
& \tilde{\omega}_{F}(t)\left(-\tilde{x}^{T}(t) P(t) b(t) u_{F}(t)+\operatorname{Proj}\left(\hat{\omega}_{F}(t), \tilde{x}^{T}(t) P(t) b(t) u_{F}(t)\right)\right) \leq 0 \\
& \tilde{\omega}_{p v}(t)\left(\tilde{x}^{T}(t) P(t) b(t) u_{f d}(t)+\operatorname{Proj}\left(\hat{\omega}_{p v}(t),-\tilde{x}^{T}(t) P(t) b(t) u_{f d}(t)\right)\right) \leq 0 \\
& \tilde{\theta}(t)\left(\tilde{x}^{T}(t) P(t) b(t)\left\|x_{t}\right\|_{L_{\infty}}+\operatorname{Proj}\left(\hat{\theta}(t),-\tilde{x}^{T}(t) P(t) b(t)\left\|x_{t}\right\|_{L_{\infty}}\right)\right) \leq 0 \\
& \tilde{\sigma}(t)\left(\tilde{x}^{T}(t) P(t) b(t)+\operatorname{Proj}\left(\hat{\sigma}(t),-\tilde{x}^{T}(t) P(t) b(t)\right)\right) \leq 0
\end{aligned}
$$

Then we have

$$
\begin{aligned}
& \dot{V}\left(\tilde{x}, \tilde{\omega}_{F}, \tilde{\omega}_{p v}, \tilde{\theta}, \tilde{\sigma}\right) \leq-\tilde{x}^{T}(t)(1-\dot{P}(t)) \tilde{x}(t)-\frac{2}{\Gamma}(\tilde{\theta}(t) \dot{\theta}(t)+\tilde{\sigma}(t) \dot{\sigma}(t)) \\
& \leq-\tilde{x}^{T}(t)(1-\dot{P}(t)) \tilde{x}(t)+\frac{2}{\Gamma}\left|\tilde{\theta}(t)^{\cdot} \theta(t)+\tilde{\sigma}(t) \cdot \sigma(t)\right|
\end{aligned}
$$

According to assumption 8,

$$
\frac{2}{\Gamma}|\tilde{\theta}(t) \cdot \theta(t)+\tilde{\sigma}(t) \cdot \sigma(t)| \leq \frac{4}{\Gamma}\left(L_{\rho} d_{\theta}+\Delta d_{\sigma}\right)
$$


which is bounded. Then according to the theorem 4.18 in [19], the error dynamics (12) is bounded as well, and the bound can be reduced by increasing the adaptive gain $\Gamma$ and $\Gamma_{F}$.

Then follow the similar argument and proving process for lemma 5.2.2 in [9], the bound in (13) is proved.

Remark 7 In contrast to the bound of (5.49) in [9], the bound of (13) is not only related to the term of $\theta_{m}()$, but also related to the uncertainty of $\omega_{F}$. Because the feedforward control signal $u_{f f}(t)$ can't pass through a low-pass filter like it does in the $L_{1}$ adaptive control, the adaptive feedforward gain $\Gamma_{F}$ can not be increased to large value due to the possible presence of noise or un-modeled high frequency dynamics in system. Otherwise, the increased adaptation gain $\Gamma_{F}$ will cause parameter drift or instability in system. Thus, the transient performance of the control law $(8) \sim(10)$ will be influenced obviously by the known disturbance and its feedforward gain uncertainty.

The lemma 1 guarantees the boundedness of system states under the control law. However, the analysis for the performance bounds of the control law is a little bit complicated. If there is no disturbance $u_{F}(t)=0$, or the known disturbance $u_{F}(t)$ could be canceled perfectly by the control signal $u_{f f}(t)=-\omega_{F} u_{F}(t)$, then the closed-loop error dynamics would be the same as that without the disturbance $u_{F}(t)$ like the (5.48) in [9]. Thus the closed-loop system under the control law would have the same performance bounds as the theorem 5.2.1 in [9].

If the known disturbance $u_{F}(t)$ would not be canceled perfectly by the control signal $u_{f f}(t)$, there are another two cases. 1). If the un-canceled part of the known disturbance $u_{F}(t)$ can be regarded as unknown disturbance, and become part of the $\sigma(t)$ satisfying the assumption on the $\sigma(t)$ in (11), then the control performance bound as that in theorem 5.2.1 in [9] could still be retained. 2). If the un-canceled part of the known disturbance $u_{F}(t)$ could not be treated as part of the unknown disturbance $\sigma(t)$, but still verify the assumption in (6), the control law would guarantee the bounded state response as stated in lemma 1.

Of course, the steady performance could not be arbitrarily improved by the increased adaptive gain $\Gamma_{F}$ as stated in theorem 5.2.1 in [9] due to the presence of disturbance $u_{F}(t)$. This is natural for a control system in practice. However, in cases when the disturbance $u_{F}(t)$ and its change rate $u_{F}(t)$ are relatively small and can be regarded as part of the unknown disturbance $\sigma(t)$, the steady-state control performance can be still guaranteed by the control law, even if the known disturbance $u_{F}(t)$ could not be canceled perfectly by the adaptive feedforward control signal $u_{f f}(t)$.

\section{The stagnation pressure control in cryogenic wind tunnel}

\subsection{Stagnation pressure dynamics with actuator dynamics and mathematical treatment}

For the design of control law for stagnation pressure, only stagnation pressure dynamics (1) will be considered here. In order to make the controller design to be more realistic in practice, the dynamics of actuator (pressure control valve) will be included here. According to the studies in reference [2, 20,21], the stagnation pressure dynamics is expressed as,

$$
P_{t}=\frac{P_{t}}{T_{t}} \cdot T_{t}+\frac{P_{t}}{W_{g}}\left(m_{L}\left(t-\tau_{1}\right)-m_{g}\left(t-\tau_{2}\right)\right)
$$




$$
\begin{aligned}
& m_{g}=\frac{k_{p v}}{\sqrt{T_{t}}}\left(\frac{P_{t}-P_{0}}{P_{0}}\right) \mu(t) \\
& \mu(s)=F(s) u(s)
\end{aligned}
$$

where $P_{t}$ is the stagnation pressure, $T_{t}$ is the stagnation temperature, and $W_{g}$ is the gas mass inside of the wind tunnel, which can be computed from the states of the tunnel. $m_{L}$ is the liquid nitrogen injection mass flow rate due to the temperature control, which is measurable. $m_{g}$ is the gas nitrogen mass flow rate extracted by the pressure control valve. $k_{p v}>0$ is a positive constant for the specific pressure control valve. $P_{0}$ is the pressure of atmosphere. $\mu(t)$ is the opening of the pressure control valve, $u(t)$ is the control signal and $F(s)$ is the partially known actuator dynamics for the pressure control valve. $\tau_{1}$ and $\tau_{2}$ are the input delays, which are quite small for pressure control in practice. Thus, the delays can be disregarded in the pressure controller design.

For the convenience of controller design, the term $m_{L}$ in (14) can be transformed into an equivalent negative gas mass extracted $-m_{g}$, and can be further transformed into equivalent negative pressure valve opening $-\mu_{F}(t)$ according to the relationship for $m_{g}$ and $\mu(t)$ in (14).

However, the pressure valve constant $k_{p v}$ in (14) is hard to be obtained precisely in practice. But it is not difficult to get the bound of $k_{p v}$ for a specific application. Thus, an additional unknown constant or slow time-varying gain factor $\omega_{p v}$ is considered in the pressure dynamics to represent the gain uncertainty. Then, the stagnation pressure dynamics with the uncertainty can be rewritten as,

$$
\begin{aligned}
& P_{t}=\frac{P_{t}}{W_{g}}\left(\omega_{F} \frac{k_{p v}}{\sqrt{T_{t}}}\left(\frac{P_{t}-P_{0}}{P_{0}}\right)\left(-\mu_{F}(t)\right)-\omega_{p v} \frac{k_{p v}}{\sqrt{T_{t}}}\left(\frac{P_{t}-P_{0}}{P_{0}}\right) \mu(t)\right)+\frac{P_{t}}{T_{t}} \cdot T_{t} \\
& \mu(s)=F(s) u(s)
\end{aligned}
$$

where $\omega_{F}$ is the gain uncertainty when transforming the $m_{L}$ into the equivalent negative pressure valve opening $-\mu_{F}(t)$. Here, an over-parameterization strategy is used. A different $\omega_{F}$ from $\omega_{p v}$ is used to represent the gain uncertainty of the transformation for $m_{L}$.

Let define

$$
b(t)=\frac{P_{t}}{W_{g}} \frac{k_{p v}}{\sqrt{T_{t}}}\left(\frac{P_{t}-P_{0}}{P_{0}}\right)
$$

and put the pressure control problem into the framework of $L_{1}$ adaptive control like that in (4), the stagnation pressure dynamics (14) can be written as

$$
\begin{aligned}
& \cdot P_{t}=A_{m} P_{t}(t)+b(t)\left(\omega_{F}\left(-\mu_{F}(t)\right)+\omega_{p v} \mu(t)+f\left(t, P_{t}(t), \sigma(t)\right)\right) P_{t}(0)=P_{t 0} \\
& \mu(s)=F(s) u(s)
\end{aligned}
$$

where $A_{m}$ is a constant representing the desired closed-loop response. Compared with (14), the extra term $A_{m} P_{t}(t)$ can be counted against by the term $f\left(t, P_{t}(t), \sigma(t)\right)$. The $\frac{P_{t}}{T_{t}}$. $T_{t}$ is regarded as an unknown disturbance in $f\left(t, P_{t}(t), \sigma(t)\right)$. The $\sigma(t)$ can account other unknown disturbances in the system. Of course, the $f\left(t, P_{t}(t), \sigma(t)\right)$ should satisfy the assumptions in section 3 .

It is assumed that the bandwidth of the actuator is much higher than the bandwidth of disturbance, or considering $F(s)=1$, then $\mu_{F}(t)=u_{F}(t)$, and $\mu(t)=u(t)$. For this reason, the control signal is expressed as 


$$
u(t)=u_{f f}(t)+u_{f d}(t)=\hat{\omega}_{F} u_{F}(t)+u_{f d}(t)
$$

where $\hat{\omega}_{F}$ is the estimate of $\omega_{F}, u_{f f}(t)$ is the feedforward control signal to cancel the known disturbance caused by $m_{L}, u_{f d}(t)$ is the feedback control signal provided by the $L_{1}$ adaptive control loop.

\subsection{Stagnation pressure controller design}

The stagnation pressure controller can be designed according to the $L_{1}$ adaptive controller defined in (8) (10) in section 3 . The parameters for the controller are set as the following. $A_{m}=-0.2, c^{T}=1$, and $P=2.5$. For an operation points of the wind tunnel, $P_{t}=200 \mathrm{kPa}$, $T_{t}=150 K, M a=0.5, k_{p v}=419.76$, and $b(t)=1.12 e 5 \times 2 . D(s)=\frac{1}{s}$, and $\mathrm{k}=0.4$. The $k_{g}(t)$ can be computed according to $k_{g}(t)=-1 /\left(c^{T} A_{m}{ }^{-1}(t) b(t)\right)$. For the adaptation law, the following projection bounds are set. $\Omega_{F}=[0.1,2], \Omega_{p v}=[0.1,4], \Theta=[-5,5]$, and $\Delta=10$. The initial values for the estimates can be chosen from the sets above. Choose $\Gamma$ and $\Gamma_{F}$ to verify $\Gamma P b(t)=1000$, and $\Gamma_{F} P b(t)=300$.

For the actuator dynamics of the pressure control valve, it is expressed as

$$
\mu(s)=F(s) u(s), F(s)=\frac{1}{0.2 s+1} e^{-0.1 s}
$$

In the simulations, the actuator dynamics has additional output saturation and rate limit. A Gaussian distributed measurement noise is added in the system output for all cases.

\subsection{Simulation results}

First, a stagnation pressure set-point change from $200 \mathrm{kPa}$ to $150 \mathrm{kPa}$ at $T_{t}=150 \mathrm{~K}$, and $M a=0.5$ of the wind tunnel is simulated. Figure 4 presents the results.

This figure shows the controller has good control performance in both transient and steady state. The tracking performance is satisfactory, and the trajectory of the total pressure can follow well the reference model associated with the state predictor (8). It is seen that the control signal is well within the rate limit of the actuator, and the actuator output can follow the control signal well.

The parameter estimates $\hat{\theta}(t), \hat{\sigma}(t)$, and $\hat{\omega}_{p v}(t)$ during the control process help achieve the desired system output response, even though these parameter estimates may not be close to their respective true values. Because there is no obvious presence of the disturbance $u_{F}(t)$, the parameter estimates for $\hat{\omega}_{F}(t)$ do not have much significance and could be switched off in this case.

Next, the adaptive feedforward control for the known disturbance $-\mu_{F}(t)$ caused by the temperature control input $m_{L}$ is tested. Figure 5 shows the total pressure control when the temperature undergoes several step changes from $150 \mathrm{~K}$ to $110 \mathrm{~K}$. The pressure setpoint is $200 \mathrm{kPa}$.

This figure shows the pressure variation during the temperature step changes. With the adaptive feedforward control, the feedforward control loop would compensate the known disturbance induced by the liquid nitrogen mass injection during temperature control more and more properly and promptly, and the pressure deviation from the set-point are getting smaller. The controller signal shows the obvious feedforward control action. In the figure, the stagnation pressure $\mathrm{Pt}$ does not converge to its 


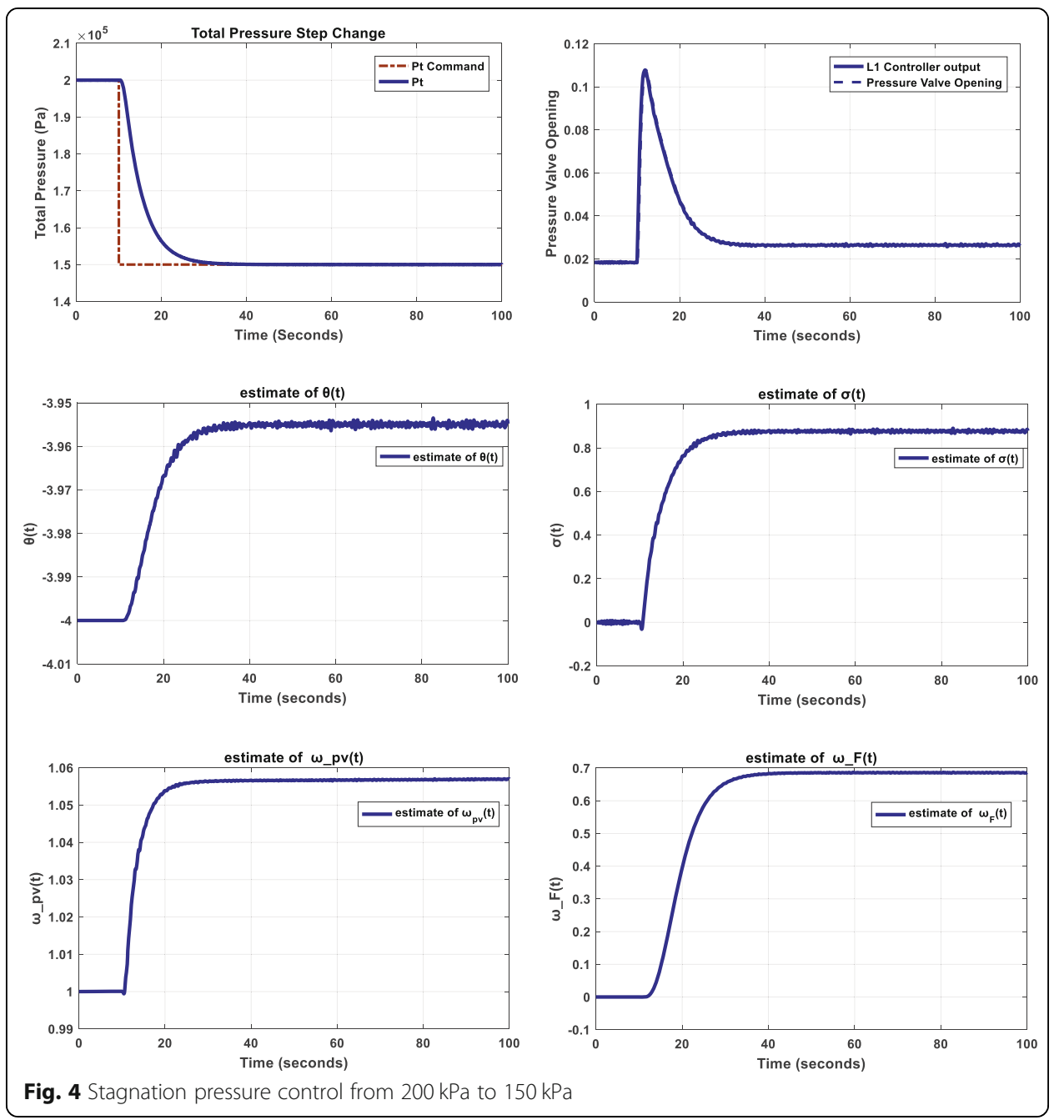

commanded value $200 \mathrm{kPa}$, and this is because that the disturbance is still presenting and changing. When the disturbance vanishes or becomes constant, the stagnation pressure Pt will settle down to its commanded value $200 \mathrm{kPa}$. Because the initial value of feedforward control gain $\hat{\omega}_{F}(t)$ is set to zero from the start, thus, even there is an obvious disturbance presenting at this moment, it is reasonable that there is no obvious feedforward compensation action in the beginning. The control action at this time is produced mainly by the $L_{1}$ adaptive feedback control loop. But with the improvement of the feedforward control gain $\hat{\omega}_{F}(t)$ estimation, the feedforward compensation action will be more prompt as the disturbance emerges.

This figure also shows the temperature step changes and the corresponding liquid nitrogen mass injection, which acts as the disturbance to the pressure control. The computed equivalent disturbance for the liquid nitrogen injection is also showed in the figure. The adaptation process will make the estimation of $\hat{\omega}_{F}(t)$ close to its true value starting from 0 . Thus, improved known-disturbance rejection could be achieved in the process.

Figure 6 gives simulation of pressure step change control from $200 \mathrm{kPa}$ to $150 \mathrm{kPa}$ during temperature step change from $150 \mathrm{~K}$ to $140 \mathrm{~K}$ at $M a=0.5$ of the wind tunnel. 

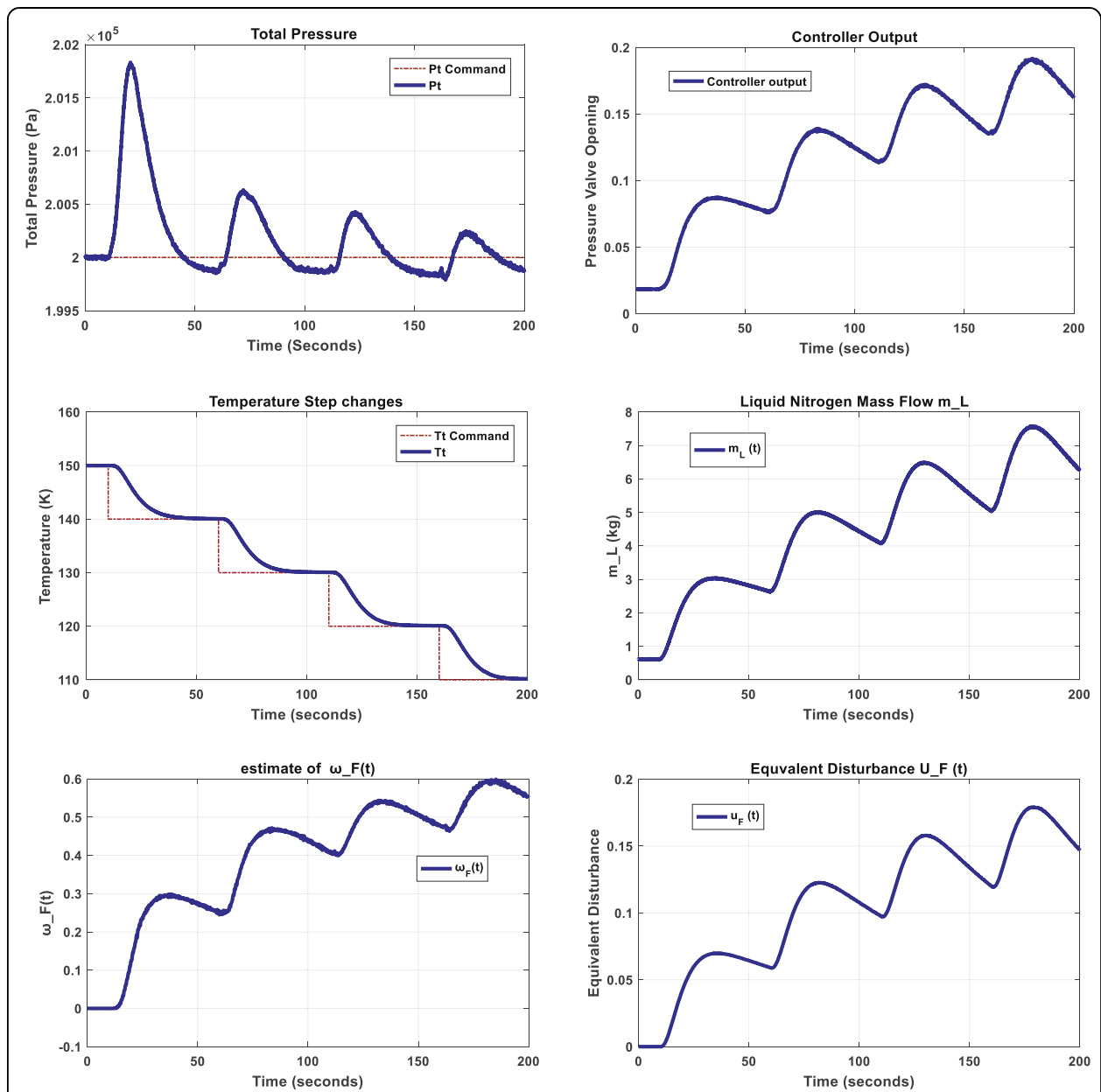

Fig. 5 Adaptive feedforward pressure control

The temperature controller output corresponds to the liquid nitrogen mass flow injection, which acts as the known disturbance to the pressure control. In this simulation, it is assumed that the feedforward control gain $\hat{\omega}_{F}(t)$ estimation has been improved after proper adaptation process. The results show that the pressure controller could compensate the disturbance caused by temperature control in the pressure control process, and the controlled pressure response is similar to that in Fig. 4, which indicates the pressure control alone without obvious temperature changes. The disturbance rejection could be observed in the controller output in the figure. Thus, the stagnation pressure controller canceled the coupled effect caused by other variable control through the nonlinear $L_{1}$ adaptive control with feedforward control action.

\section{Conclusion}

By introducing an adaptive feedforward control loop to the standard $L_{1}$ adaptive control structure, a nonlinear $L_{1}$ adaptive controller with adaptive feedforward control action is proposed in this paper. After some stability analysis and discussion, it is shown that this new control method could effectively compensate known bounded 

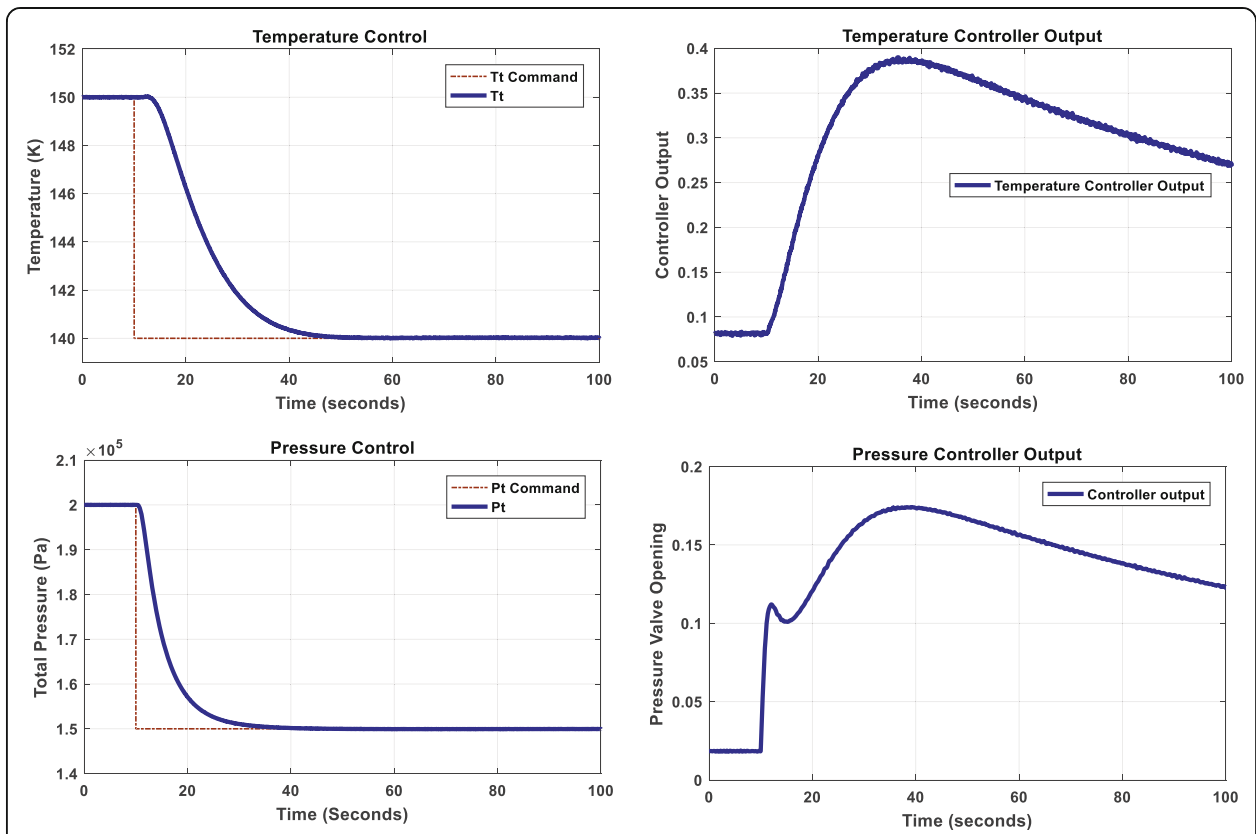

Fig. 6 Pressure step change control during the temperature step change

disturbances with linear gain uncertainty, while retaining the basic feature of $L_{1}$ adaptive control. The application in the stagnation pressure control in cryogenic wind tunnel and the simulation results have verified the effectiveness of this new control architecture. The proposed control approach, with the ability of canceling coupled effects in Multi-input \& Multi-output control process, could be also used in the control for temperature and Mach number in cryogenic wind tunnel.

\section{Abbreviations}

atm: Atmospheric pressure; BIBO: Bounded-input-bounded-output; DC: Direct current (Here it mainly indicates the low frequency range near zero); $G N_{2}$ : Gas nitrogen; $K$ : Kelvin (Temperature unit); $L_{1}: L_{1}$ norm of signal in math; $L N_{2}$ : Liquid nitrogen; PI: Proportional-Integration; $L_{\infty}: L_{\infty}$ norm of signal in math

\section{Acknowledgements}

Not applicable

\section{Authors' contributions}

RZ proposed the research idea, completed the research and wrote the main body of this article. MX helped implement the simulation and write this article. DL helped propose the research topic and revise this article. GT analyzed the simulation results and offered his view on the performance of the method in this article. WG analyzed part of the simulation results. All authors read and approved the final manuscript.

\section{Funding}

Not applicable

\section{Availability of data and materials}

Not applicable

\section{Competing interests}

The authors declare that they have no competing interests.

Received: 11 September 2019 Accepted: 13 November 2019

Published online: 02 January 2020

\section{References}

1. Goodyer MJ, Kilgore RA (1973) High-Reynolds-number cryogenic wind tunnel. AIAA J 11(5):613-619. https://doi.org/10. $2514 / 3.50500$ 
2. Balakrishna S, Kilgore W, Thibodeaux J (1992) "Control of large cryogenic tunnels," 28th Joint Propulsion Conference and Exhibit. American Institute of Aeronautics and Astronautics

3. Armstrong ES, Tripp JS, Aeronautics USN, Scientific SA, Branch TI, Center LR (1981) An application of multivariable design techniques to the control of the National Transonic Facility: National Aeronautics and Space Administration, Scientific and Technical Information Branch

4. Gobert J (1994) "ETW control system - design and first results," 25th Plasmadynamics and Lasers Conference. American Institute of Aeronautics and Astronautics

5. Paryz R (2011) "Recent developments at the NASA Langley research center national transonic facility," 49th AIAA Aerospace Sciences Meeting including the New Horizons Forum and Aerospace Exposition, p 877

6. Balakrishna S (1989) Microcomputer based controller for the Langley 0.3-meter Transonic Cryogenic Tunnel. National Aeronautics and Space Administration, Langley Research Center. National Technical Information Service, distributor, Hampton, Springfield

7. Tripp JS, Aeronautics USN, Scientific SA, Branch TI (1983) Development of a distributed-parameter mathematical model for simulation of cryogenic wind tunnels: National Aeronautics and Space Administration, Scientific and Technical Information Branch

8. Paryz R (2012) "Upgrades at the NASA Langley Research Center National Transonic Facility," 50th AIAA Aerospace Sciences Meeting including the New Horizons Forum and Aerospace Exposition. American Institute of Aeronautics and Astronautics

9. Hovakimyan N, Cao C (2010) L $L_{1}$ Adaptive Control Theory: Guaranteed Robustness with Fast Adaptation: SIAM

10. Aström K (2014) History of adaptive control. In: Encyclopedia of Systems and Control. Springer London, London, pp 1-9

11. Astrom KJ (1987) Adaptive feedback control. Proc IEEE 75(2):185-217. https://doi.org/10.1109/PROC.1987.13721

12. Wise KA, Lavretsky E, Hovakimyan N (2006) Adaptive control of flight: theory, applications, and open problems, 2006 American Control Conference. IEEE, p 6

13. Steinberg M (2005) Historical overview of research in reconfigurable flight control, Proceedings of the Institution of Mechanical Engineers, Part G. J Aerospace Eng 219(4):263-275. https://doi.org/10.1243/095441005×30379

14. Cao C, Hovakimyan N (2006) Design and analysis of a novel $L_{1}$ adaptive controller, Part I: Control signal and asymptotic stability, American Control Conference, 2006. IEEE, pp 3397-3402

15. Cao C, Hovakimyan N (2006) Design and analysis of a novel $L_{1}$ adaptive controller, part II: Guaranteed transient performance, American Control Conference, 2006. IEEE, pp 3403-3408

16. Cao C, Hovakimyan N (2007) Guaranteed transient performance with $L_{1}$ adaptive controller for systems with unknown time-varying parameters and bounded disturbances: Part I, American Control Conference, 2007. ACC'07. IEEE, pp 39253930

17. Cao C, Hovakimyan N (2007) Stability margins of $L_{1}$ adaptive controller: Part II, American Control Conference, 2007. ACC'07. IEEE, pp 3931-3936

18. Pomet JB, Praly L (1992) Adaptive nonlinear regulation: estimation from the Lyapunov equation. IEEE Trans Autom Control 37(6):729-740. https://doi.org/10.1109/9.256328

19. Khalil HK (2002) Nonlinear Systems. Prentice Hall, New York

20. Zhu R, Yin G, Tang G, Wang H, Zhang S (2018) Temperature trajectory control of cryogenic wind tunnel with robust $L_{1}$ adaptive control. Trans Inst Meas Control 40(13):3675-3689. https://doi.org/10.1177/0142331217728569

21. Zhu R, Yin G, Chen Z, Zhang S, Guo Z (2018) Temperature control of cryogenic wind tunnel with a modified $L_{1}$ adaptive output feedback control. Measurement Contr 51(9-10):498-513. https://doi.org/10.1177/0020294018808672

\section{Publisher's Note}

Springer Nature remains neutral with regard to jurisdictional claims in published maps and institutional affiliations.

\section{Submit your manuscript to a SpringerOpen ${ }^{\circ}$ journal and benefit from:}

- Convenient online submission

Rigorous peer review

- Open access: articles freely available online

- High visibility within the field

- Retaining the copyright to your article

Submit your next manuscript at $\boldsymbol{\nabla}$ springeropen.com 